\title{
Prácticas pedagógicas para la inclusión en dos modelos educativos alternativos*
}

\author{
DOI: https://doi.org/10.18046/recs.i34.4243 \\ Pedagogical Practices for Inclusion in Two \\ Alternative Educational Models \\ Jackeline Cantor ${ }^{* *}$ \\ Universidad Icesi (Cali, Colombia) \\ José Eduardo Sánchez ${ }^{* * *}$ \\ Universidad Icesi (Cali, Colombia) \\ Danna Aristizábal-Oviedo ${ }^{* * * *}$ \\ Universidade de Brasília (Brasilia, Brasil)
}

\begin{abstract}
* El artículo se deriva del proyecto de investigación "Estrategias pedagógicas alternativas para el fortalecimiento y la calidad educativa en instituciones de la ciudad de Cali". Fue financiado en el marco de la convocatoria interna de la Universidad Icesi 2016. La investigación fue concluida en el 2017. Artículo de investigación recibido el 19.08.2020 y aceptado el 12.01.2021.

** Psicóloga y Magister en Psicología de la Universidad del Valle (Colombia). Profesora del Departamento de Estudios Psicológicos de la Universidad Icesi (Colombia). Correo electrónico: jcantor@icesi.edu.co ORCID: https://orcid. org/oooo-0oo2-6339-8701

*** Psicólogo y Magíster en Psicología de la Universidad del Valle (Colombia), doctorando Universitat de Girona (España). Profesor del Departamento de Estudios Psicológicos de la Universidad Icesi (Colombia).Correo electrónico:jesanchez@ icesi.edu.co ORCID: https://orcid.org/oooo-ooo2-4580-1894

**** Psicóloga, Máster en psicología, doctoranda de la Universidad de Brasilia (Brasil). Correo electrónico: dannaaristizabal@gmail.com ORCID: https://orcid.org/oooo-ooo1-9982-3047
\end{abstract}




\section{Cómo citar/How to cite}

Cantor, Jackeline; Sánchez, José Eduardo; Aristizábal-Oviedo, Danna (2021). Prácticas pedagógicas para la inclusión en dos modelos educativos alternativos. Revista CS, 34, 43-69. https://doi.org/10.18046/recs.i34.4243 


\section{Resumen}

El presente estudio exploró la relación entre las prácticas docentes y los niveles percibidos de inclusión por maestros y estudiantes en dos modelos pedagógicos alternativos. Se midieron elementos constitutivos de los modelos que teóricamente parecen favorecer la inclusión a través de encuestas a docentes y estudiantes, y se hicieron pruebas de correlación para comprobar su asociación con la percepción de inclusión. Los resultados muestran diferencias entre los reportes de docentes y estudiantes. Para los primeros, las prácticas centradas en los sujetos, pertinentes al contexto y con incidencia comunitaria a favor de la organización social, ostentan mayores puntajes de inclusión que las de tipo dialógico; mientras que segundos otorgan más puntaje a estas últimas. Esto sugiere que las prácticas centradas en promover lo dialógico entre individuos resultan más efectivas desde la perspectiva de los estudiantes en términos de generar inclusión en el aula.

\section{PALABRAS CLAVE:}

educación, prácticas pedagógicas, prácticas dialógicas, inclusión educativa, inclusión escolar

In the present study, the relationship between teaching practices and perceived levels of inclusion was explored for both teachers and students in two alternative pedagogical models. Constitutive elements of the models, that theoretically seem to favor inclusion, were measured through surveys of teachers and students, and correlation tests were carried out to verify their association with the perception of inclusion. The results show differences in the reports given by teachers and students. For teachers, the practices centered on the subjects, pertinent to the context and with community incidence in favor of social organization present higher inclusion scores than the dialogic-type practices, while, for the students, dialogic-type practices present higher scores. These results suggest that practices focused on promoting dialogue between individuals are more effective from the students' perspective in terms of fostering inclusion in the classroom.

\section{KEYWORDS:}

Education, Pedagogical Practices, Dialogic Practices, Educational Inclusion, School Inclusion 



\section{Introducción}

De acuerdo con la Organización de las Naciones Unidas para la Educación, la Ciencia y la Cultura - Unesco (2008; 2017) la inclusión escolar es el proceso que asegura el acceso a ella no solo a quienes se han pensado históricamente como excluidos - es decir, los que presentan una situación de discapacidad, o estudiantes que se han denominado con necesidades especiales - , sino también a todos los estudiantes que puedan ser sujetos susceptibles de ser excluidos. De manera similar, Valdés, López y Jiménez (2019) plantean que un desafío para la educación inclusiva es reducir la exclusión del sistema educativo en pro de asegurar el proceso de participación, aprendizaje y acceso a todos los estudiantes, incluso a los más vulnerables. En este sentido, el concepto de inclusión en la educación va más allá de reconocer a los aprendices con capacidades distintas: implica generar espacios que promuevan una convivencia efectiva, entendida esta como la posibilidad de oportunidades para todos (Arias et al., 2007). Durante las últimas décadas, la política pública educativa colombiana ha concentrado sus esfuerzos alrededor de la calidad y la inclusión; ejemplo de ello es la promulgación de leyes ${ }^{1}$ que buscan articular la calidad educativa, entre otros factores, con la generación de condiciones de inclusión escolar.

El aumento de la cobertura escolar incrementa la pluridiversidad entre los estudiantes en los ambientes escolares en tanto reúne diferencias étnicas, de género, religiosas, culturales y sociales, y de aprendizaje, así como personas con discapacidad. De forma paradójica, la diversidad también puede estimular y reproducir situaciones de discriminación y segregación. En este sentido, la escuela constituye un espacio de encuentro de diversas acciones educativas encaminadas a mejorar las relaciones humanas, resolver los conflictos, prevenir la violencia y el fracaso escolar. Así, se promueven prácticas de convivencia escolar que, a su vez, posibilitan el acceso y la permanencia en la escuela (Valdés et al., 2019).

De acuerdo con Ainscow, Booth y Dyson (2006), la educación inclusiva es un proceso continuo de mejora e innovación sistemático, enfocado a promover el aprendizaje, la participación y la integración entre los estudiantes, con especial atención a quienes puedan estar en condiciones más vulnerables. En un sentido similar, Booth, Nes y Stromstad (2003) señalan algunos matices asociados al concepto de inclusión: la diversidad de los estudiantes, la superación de las barreras para el aprendizaje y la estimulación de la participación de docentes, estudiantes y otros actores de la comunidad educativa. Así, se resalta que la inclusión tiene que ver con la ausencia

1. Destaca la Ley 1618 (2013), en la que se consideran las disposiciones necesarias para el ejercicio de los derechos de las personas con discapacidad y el Decreto 1421 (2017), que reglamenta lo dispuesto por la ley en lo concerniente a la inclusión educativa de las personas con discapacidad. 
de formas de exclusión; por tanto, un sistema educativo inclusivo sería aquel en donde el sujeto fuera reconocido y se sintiera parte de la comunidad. De ahí que sea necesario el trabajo colaborativo entre los diversos actores que configuran las comunidades educativas e instituciones (Booth; Ainscow, 2002).

En este sentido, el presente artículo muestra los resultados parciales de una investigación adelantada en tres instituciones educativas de la ciudad de Santiago de Cali (Depto. del Valle del Cauca, Colombia), en la que se exploraron sus modelos educativos (o algunas prácticas) que propenden a la mejora en la calidad educativa y la inclusión. En particular, se abordó la relación entre las prácticas docentes y los niveles percibidos de inclusión por maestros y estudiantes en dos modelos pedagógicos alternativos. Para ello, y de acuerdo con las propuestas de cada modelo, se midieron los elementos constitutivos que, en teoría, buscan favorecer la inclusión a través de encuestas a docentes y estudiantes, y se hicieron pruebas de correlación para comprobar su asociación con la percepción de inclusión.

\section{La inclusión educativa: contexto y momento actual}

En tanto concepto, la educación inclusiva ha atravesado un proceso de tipo evolutivo que inició en la década de 1950, momento en que apareció la educación especial -entendida inicialmente desde la perspectiva de la integración escolar-, hasta la actualidad, en la que se considera la existencia de un aprendiz diverso (Beltrán; Martínez; Vargas, 2015; Parra, 1996). Este proceso histórico incluye la formulación de conceptos clave, uno de los cuales corresponde a las necesidades educativas especiales (NEE): esto ponía el acento en determinar, a través de un diagnóstico, las características diferentes de algunos estudiantes a partir de las cuales eran ubicados en grupos escolares particulares. Las NEE avanzan sin superar por completo las perspectivas de la integración escolar, que tuvieron un importante desarrollo en la escolaridad durante la década de 1980. La concepción de la propuesta de educación integradora comenzó a virar hacia la inclusión luego de la "Declaración de Salamanca" de 1994, momento en que empezó a transformarse en educación inclusiva, la cual busca crear sistemas educativos que retomen la diversidad y consideren la adaptación de las propuestas pedagógicas y didácticas de acuerdo con ella. Según Arias (2014) esta propuesta del aprendiz diverso no es asumida de una manera fácil por todos los docentes: para algunos de ellos, las formas tradicionales de educación perseveran y el paradigma de integración y de educación especial aparece en sus formatos de clase de manera recurrente.

En Estados Unidos y Europa, y de acuerdo con Vrasmas (2018), los académicos han entendido la educación inclusiva como un complejo y controversial concepto 
que apunta a la reorganización y cambio de los sistemas educativos. Desde una primera aproximación al concepto, históricamente la inclusión escolar como teoría y práctica en las instituciones educativas comienza a principios de la década de 1980 en Europa y Estados Unidos, como una iniciativa orientada hacia estudiantes con discapacidad (Fuchs; Fuchs, 1994; Lipsky; Gartner, 1996). No obstante, con el viraje social y político de las últimas décadas, la dirección de esta práctica se ha trasformado en un auténtico reto al buscar que todas las personas tengan acceso efectivo a prácticas inclusivas: es un desafío en tanto cuestiona directrices normativas previas sobre la mejor manera de enseñar y de aprender, al buscar responder a las distintas necesidades que pueden tener los diversos estudiantes en los escenarios escolares por sus características étnicas, de género, lenguaje o nacionalidad, entre otras (Infante, 2010). En este sentido, la inclusión educativa constituye una forma de respuesta y abordaje de la diversidad en los contextos escolares; da lugar al reconocimiento y a la representación de los distintos sujetos susceptibles de ser interpelados, y abre el camino a las distintas subjetividades posibles.

De modo general, y nuevamente siguiendo a Vrasmas (2018), el abordaje de la educación inclusiva desde la academia ha tenido tres momentos. El primero, relacionado con la noción del mejor ambiente escolar, tuvo lugar a comienzos de la década de 1990: se utilizaron entonces la investigación y los estudios alrededor de esta temática como base para sustentar la necesidad de la reconstrucción de prácticas y políticas educativas. En el segundo periodo, en el año 200o, la investigación se caracterizó por buscar que la educación respondiera a las necesidades de cada estudiante. En el tercero, que puede ser considerado actual, los propósitos de las investigaciones han girado en torno a la reconstrucción de este campo dentro de las ciencias de la educación, de acuerdo con valores y principios del enfoque inclusivo, y con el objetivo de lograr soluciones multidisciplinarias y desarrollos de sistemas educativos dimensionados en una pedagogía de la inclusión (Vrasmas, 2018).

El concepto de educación inclusiva en los estudios actuales se introduce con el objetivo de recomendar soluciones encaminadas al desarrollo de nuevas actitudes y percepciones sociales, que permitan la implementación efectiva de la inclusión en contextos escolares (Allman, 2013; Burgstahler, 2012; Clavijo; Bautista, 202O; Ferguson, 2008; Florian, 2014; Lewis; Norwich, 2005). En la actualidad, la investigación sobre la inclusión escolar no solo busca y propone soluciones para casos concretos; también promueve la multidisciplinariedad del campo (Allman, 2013) y el análisis y conformación de nuevos conceptos, así como la reformulación y redefinición de los conceptos principales como inclusión escolar o educativa, educación inclusiva e inclusión social. Con esto, otra característica de la educación inclusiva es que constituye un proceso continuo de adaptación de la escuela y los maestros en cuanto al 
reconocimiento de la diversidad de los aprendices y sus necesidades individuales y sociales, lo que solo es posible en tanto se generen espacios para la construcción de políticas, prácticas y la transformación del ethos institucional (Clavijo; Bautista, 2020; Vrasmas, 2018).

Echeita y Duk (2008) apuntan a una característica clave de la educación de la región: la preocupación inicial por la inclusión en Iberoamérica es consecuencia de los altos niveles de exclusión, desigualdad educativa y acceso real a posibilidades y recursos. Estos autores destacan en su investigación la necesidad de considerar el abordaje de la inclusión en contextos escolares desde una perspectiva que adopta una mirada contextual de la región: la educación inclusiva en Iberoamérica, y especialmente en Colombia, debe considerar en su aplicación y desarrollo las condiciones que han marcado las trayectorias sociales de los distintos contextos, esto es, encontrar esas particularidades que han construido, reforzado y reproducido tales subjetividades. En este sentido, los autores plantean que es necesario tener presente la consideración de que, en sociedades desiguales, la calidad de los aprendizajes tiene estrecha relación con el contexto de producción de ese aprendizaje.

De lo anterior se considera que las escuelas valiosas no son solo las que logran indicadores esperados en las pruebas y mediciones estandarizadas; por el contrario, también resulta crucial reconocer instituciones educativas que contribuyan a compensar las diferencias y condiciones de origen, o que generen más valor agregado a la realidad social de sus estudiantes (Echeita; Duck, 2008). En este sentido, la inclusión educativa permite no solo la inclusión individual de los estudiantes, sino de la escuela misma, en tanto posibilita el cambio de la perspectiva sobre los procesos de enseñanza y aprendizaje, en los que la misma escuela es un sujeto susceptible de ser transformado y permeado por los sujetos y contextos sociales que cohabitan. Con esto, se requieren propuestas que apunten a incrementar, en docentes, estudiantes y agentes comunitarios, prácticas que abran camino a ambientes escolares inclusivos. Diversas investigaciones y experiencias de intervención (Bravo; Santos, 2019; Cantor, 2018; Echeverría; Flórez, 2018; Sánchez, 2018) muestran la importancia de identificar las percepciones, concepciones, teorías implícitas o representaciones sociales, que orientan las prácticas docentes, pues éstas se constituyen en un eje articulador y promotor de pensamiento reflexivo y transformador de las prácticas interactivas.

\section{Perspectivas metodológicas orientadas a la inclusión}

De acuerdo con Guerrero (2012), varios estudios consideran que las estrategias y metodologías inclusivas son condiciones necesarias para lograr llevar a cabo una educación incluyente (Ainscow, Soutworth; Hopkins; West, 2001; Arnaiz, 2011; 
Echeita; Duck, 2008; Mateus; Vallejo; Obando; Fonseca, 2017). De una forma más precisa, se podría plantear que la inclusión educativa, como elemento central de la educación que se debe impartir en cualquier institución escolar, debe propiciar un cumplimiento de la misión de la educación, en lo que respecta a hacerla cada vez más pertinente frente al contexto actual de las necesidades y posibilidades de los estudiantes. La pertinencia estaría dada en la medida en que la escuela es eficaz, lo que de acuerdo con Ainscow et al. (2001:14) supone "La decisión de hacer algo para mejorar la eficacia escolar se basa en aquello que más les conviene a los alumnos". Ahora bien, en pro de este objetivo, los autores plantean que es necesario generar metodologías participativas y reflexivas como elemento clave para que se dé una apuesta educativa inclusiva que se traduzca en mejoras institucionales.

En la misma línea, Mateus et al. (2017) plantean la importancia de reconocer las percepciones que sobre la cultura y las prácticas inclusivas tienen los diferentes estamentos de la comunidad, en pro de una educación inclusiva real. Al respecto, Arnaiz (2011) propone que el establecimiento de estrategias y metodologías acordes a la educación inclusiva es el primer paso para que se promuevan los procesos de una propuesta participativa y significativa. Gran parte de estas estrategias y recursos están cimentados en enfoques constructivistas; estos buscan un aprendizaje significativo, cuyo objetivo es potenciar de forma permanente el aprendizaje y la autonomía por medio de la acción y la experimentación.

Este enfoque constructivista enfatiza procesos cognitivos, sociales y afectivos de la conducta mediante un proceso de interacción del ambiente escolar y de los docentes, estudiantes y la comunidad escolar en general. Desde la propuesta de educación inclusiva, que centra su trabajo en el DUA (Diseño Universal de Aprendizaje ${ }^{2}$ ), es fundamental partir del reconocimiento de las características de los estudiantes y de los modos en que ellos aprenden involucrando a la comunidad educativa y desarrollar así las propuestas curriculares inclusivas que se proponen desde el Estado.

El Ministerio de Educación Nacional colombiano (MEN, 2009: 25) define la inclusión como "la posibilidad de que todas las personas se formen y eduquen en la institución educativa de su sector y puedan gozar de todos los recursos que tiene esta, sin que se le discrimine o limite su participación". Esto es posible solo si las instituciones generan lo que el MEN plantea respecto a crear acciones inclusivas que promuevan, implementen, planeen, realicen seguimiento y evaluación a los procesos curriculares, con el objetivo de "atender con calidad y equidad las necesidades en el aprendizaje, la participación y la convivencia de su comunidad educativa" (MEN, 2009:24). En este sentido, cada institución educativa debe considerar en su proyecto

2. Enfoque desarrollado por el Center for Applied Special Technology (Centro de Tecnología Especial Aplicada - CAST). 
educativo institucional (PEI) los siguientes aspectos: accesibilidad, participación, evaluación flexible, resolución de conflictos, prevención del fracaso escolar, participación de los padres de familia, entre otros, lo que propendería a la promoción de una cultura y de prácticas inclusivas (MEN, 2009).

Ahora bien, dada la naturaleza del enfoque inclusivo, en las metodologías deben primar los procesos activos, constructivos que incentiven la participación de todos desde la diferencia, y esta sea tenida en cuenta para potenciar procesos de trabajo en el salón de clase a nivel cognitivo, afectivo y social. Dentro de la propuesta inclusiva se hace prioritario plantear criterios y procedimientos que generen una real flexibilidad para la evaluación de cada uno de los estudiantes y la promoción escolar; todo esto sin dejar de lado la necesidad de crear servicios de apoyo y asesoramiento orientados a la globalidad de los centros e instituciones educativas (Guerrero, 2012).

De acuerdo con Guerrero (2012), las diversas metodologías se clasifican, grosso modo, de la siguiente manera:

- Metodologías y técnicas de aprendizaje cooperativo: tutoría entre pares, grupos de apoyo, actividades.

- Metodologías y técnicas de aprendizaje colaborativo: en ellas, la propuesta central es un trabajo de apoyo y de colaboración entre profesores y pares. Formación a partir de la reflexión de situaciones o casos que correspondan al contexto de la escuela. Aprendizaje basado en problemas, investigación - acción - reflexión., etc.

- Metodologías y técnicas experienciales transformativas: la opción que presenta más fuerza en esta metodología es de carácter experiencial, en el cual todo lo que se vivencie en el trabajo escolar es motivo de análisis grupal e individual. La forma de reflexión que se propone se corresponde en algunas ocasiones con la forma en que Freire plantea una pedagogía liberadora.

- Técnicas de evaluación del aprendizaje colaborativo/cooperativo/experiencial: en este punto aparecen formas de evaluación que han sido usadas siempre en la escuela como las pruebas orales y escritas, pero también se propone generar espacios dialógicos en los cuales circule la voz de los estudiantes. La rúbrica, así como otros elementos cualitativos para la evaluación, aparecen como importantes, desde el lugar de que no solo el docente los conoce, sino que el estudiante mismo puede partir de ellos a fin de pensar su propia actuación educativa (Guerrero, 2012). 


\section{Comunidades de Aprendizaje y el Modelo Comunitario de Oriente como apuesta por la inclusión}

Este estudio presenta dos estrategias socioeducativas encaminadas a mejorar procesos educativos escolares: comunidades de aprendizaje y el Modelo Educativo Comunitario de Oriente. De acuerdo con Sánchez (2018), el proyecto de Comunidades de Aprendizaje se viene implementando hace varios años en Colombia. Su propuesta, basada en el aprendizaje dialógico, cuestiona las perspectivas tradicionales basadas en la concepción del aprendizaje como transmisión de conocimiento. El aprendizaje dialógico asume una perspectiva social y constructivista del conocimiento, de acuerdo con la que el aprendizaje se produce en las interacciones. En este sentido, se promueve la participación activa de los estudiantes en su aprendizaje y la transformación del rol docente (Sánchez, 2018).

El modelo Comunidades de Aprendizaje, desarrollado por el Centro Especial de Investigación en Teoría y Prácticas Superadoras de la Desigualdad (CREA) de la Universidad de Barcelona (Aubert; Flecha; García; Flecha; Racionero, 2008), propone una forma novedosa de gestionar los contextos educativos a través de la búsqueda de la transformación de las instituciones educativas en comunidades que den cabida a todos los miembros del entorno social, para así promover mejoras en el aprendizaje y, especialmente, en la convivencia. Este modelo propone considerar la participación de la comunidad como eje central en las actuaciones educativas de éxito y las interacciones dialógicas entre padres de familia y los demás miembros de la comunidad. Algunos estudios, que han explorado la efectividad de estas prácticas en relación con el desempeño académico de los estudiantes de básica primaria (Díez; Flecha 2010) y las condiciones de convivencia (Flecha; García; Gómez; Latorre, 2009), indican mejoras en los desempeños de los estudiantes a través del tiempo y en las relaciones que existen entre escuela y comunidad. Así, dado que el modelo Comunidades de Aprendizaje ubica como eje central la participación activa de la comunidad educativa (Valdés et al., 2019), se esperaría que sus prácticas promovieran la inclusión en el contexto educativo.

Por su parte, el Modelo Educativo Comunitario de Oriente es producto de una propuesta estatal surgida en el 2011; propone un énfasis en el arte y la cultura, potenciando la expresión del sentir y pensar de los estudiantes. Se enfoca en la comprensión y transformación de esquemas de representación de la realidad del estudiante a través de la práctica ejercida por el docente (Institución Educativa Nuevo Latir, 2011). Aquí, primero, el docente reconoce los esquemas de percepción específicos de los estudiantes, determina su capital cultural y la manera como estos orientan las prácticas. Segundo, plantea una ruptura entre las representaciones y prácticas, 
con el fin de transformarlas. Se trata de un proceso de recontextualización (Bernstein, 1990) en que el estudiante es considerado un sujeto activo que cuenta con la posibilidad de construir y reconstruir su entorno a partir de las herramientas que la interacción con otros agentes le provee, siempre en función de las demandas de su contexto. De esta manera, el Modelo Educativo Comunitario de Oriente promueve el reconocimiento de las particularidades de los individuos como eje articulador de las prácticas educativas; es decir, favorece la inclusión.

Estas dos propuestas introducen dos cuestiones fundamentales en el proceso de la educación inclusiva. Por un lado, abren la posibilidad al intercambio y escucha de significados propios sobre temas concretos, creando una situación de diálogo en que estudiantes, acudientes y demás integrantes de la comunidad educativa interactúan, con lo que se posibilita el intercambio de reflexiones individuales que son recogidas y validadas. Esto es importante porque el diálogo igualitario permite un cambio de roles que los promueve como agentes educativos (Cantor, 2018). De otro lado, las tertulias dialógicas son otra práctica educativa que devuelve la responsabilidad del aprendizaje al estudiante, al introducir situaciones donde este es quien desea aprender, de tal forma que el proceso educativo en el aula se transforma en un espacio dinámico y de interacción constante con el otro. Además de esto, se considera importante mencionar que la comprensión de una pedagogía incluyente va más allá de la descripción de métodos de enseñanza; su entendimiento debe pasar por algunas consideraciones a tener en cuenta en la construcción de modelos, tales como la compleja relación entre los métodos que los profesores prefieren, las distintas identidades, actitudes y deseos de los alumnos, las circunstancias especiales del contexto y las adaptaciones en cuanto a valores y contenido del currículo.

Con todo lo planteado, se considera que la inclusión educativa es un proceso gradual de trasformaciones y cambios que apunta a eliminar las barreras que causan exclusiones en el sistema educativo. Por lo tanto, no solo visibiliza la atención sobre las capacidades diferentes en el aula; también reconoce y posibilita las distintas maneras de ser y estar dentro del tejido social, de tal forma que se constituya un camino hacia una sociedad más inclusiva.

\section{Metodología}

\section{Participantes y contexto de recolección}

Se recolectó información en tres instituciones educativas (IE) que implementan alguno de los modelos estudiados; 2 con Comunidades de Aprendizaje y 1 con el Modelo Educativo Comunitario de Oriente. 
Todas las IE estudiadas tienen carácter público. Las dos que implementan la estrategia de Comunidades de Aprendizaje están ubicadas en la zona de ladera de la ciudad que, al igual que el distrito de Aguablanca donde está ubicada la IE que desarrolla el modelo Educativo Comunitario de Oriente, son zonas de habitación de personas de clase popular. En ellas existen diversas problemáticas sociales con múltiples causas y manifestaciones, tales como exclusión, altos índices de violencia, desempleo, empleo informal y problemas de salud, entre otras.

Se usó un muestreo no probabilístico por conveniencia. La muestra de docentes estuvo conformada por 7 participantes: 3 para Comunidades de aprendizaje (CA) y 4 para el Modelo Educativo Comunitario de Oriente. Los primeros estaban encargados de los grados tercero (2) y quinto de primaria (1); y en cuanto a los segundos, 2 se encargaban de grado tercero y 2 de quinto. Los docentes participantes eran todos licenciados con más de cuatro años de ejercicio docente y su participación fue voluntaria. Se seleccionaron docentes de los grados $3 .^{\circ}$ y $5 .{ }^{\circ}$ de primaria porque en estos tiene lugar la evaluación estatal estandarizada; al respecto, existía un interés especial de los docentes y de las IE por conocer la perspectiva de los estudiantes sobre la calidad y la inclusión educativas. La característica de la cantidad de integrantes de la muestra marca una pauta importante sobre el tipo de análisis que se realizaron con los datos provenientes de esta. Esto es, al ser una muestra con un $\mathrm{N}$ reducido, es solo considerable para presentación de datos estadísticos descriptivos. No obstante, esto no constituye una limitación de la investigación porque esta no busca una medida de representatividad en promedio de la población, sino $-\mathrm{y}$ de modo esencial- la identificación de los elementos prácticos asociados a la educación inclusiva de los docentes encargados en grados académicos de instituciones educativas seleccionadas de forma intencional. La validez de la comparación entre los reportes de los docentes y los estudiantes recae sobre el rol que ocupa cada uno en la aplicación del modelo y estrategia pedagógica, y no en las consideraciones sobre tamaño del efecto y generalización.

Como una estrategia de confiabilidad de las medidas, se decidió indagar en las percepciones de los estudiantes sobre las prácticas de sus docentes. Así, se incluyeron en la investigación 164 estudiantes, 75 de ellos pertenecientes al modelo Comunidades de Aprendizaje ( 37 en el grado $3 .^{\circ}$ y 30 en $5 .^{\circ}$ ) y 89 pertenecientes al Modelo Educativo Comunitario de Oriente ( 40 en grado $3 .^{\circ} \mathrm{y} 49$ en $\left.5 .^{\circ}\right)$.

\section{Instrumento}

Se diseñaron dos encuestas tipo Likert con escalas de 1 a 5 , dirigidas a docentes y estudiantes de los grados $3 .^{\circ}$ y $5 .^{\circ}$ de básica primaria, en las que se indagó por sus 
prácticas en relación con los siguientes elementos que responden a aspectos constitutivos de los modelos pedagógicos:

- Componente dialógico: consiste en dar lugar al estudiante en relación con su contexto, su historia, sus intereses y necesidades.

- Formación de agentes políticos: promover la formación crítica y la toma de postura en los estudiantes.

- Habitancia: promover relaciones interpersonales y con el entorno equilibradas

- Aprendizaje colaborativo: asignación de objetivos y tareas en función de las capacidades diferenciales.

- Arte: uso del arte como herramienta para la formación dialógica, dada en actividades como la danza, la pintura y la música.

- Alineación de objetivos: plantear objetivos de la escuela que respondan a las necesidades de la comunidad.

- Integración de campos de conocimiento: integrar temas de diferentes dominios de conocimiento en una clase.

- Tecnologías de la Información y la Comunicación (TIC): usar estas últimas para dar cabida a los distintos miembros del entorno social, la construcción colectiva del conocimiento y el reconocimiento de la diversidad de los educandos.

A cada indicador se asociaron tres ítems. Así, la encuesta constó de 39 ítems para los docentes y 36 para los estudiantes. La diferencia corresponde a la característica de articulación del proceso de enseñanza y las exigencias del MEN, que resultaba solo pertinente para los docentes. El cuadro 1 muestra los detalles de las variables, definiciones e indicadores asociados.

Para la validación del instrumento, tres profesores del área de psicología educativa vinculados con la investigación, pero que no habían formado parte de la formulación de las preguntas, revisaron el contenido de los ítems para establecer: a) claridad; b) redacción; c) si contiene información necesaria y suficiente para responder adecuadamente; y d), si la complejidad es adecuada respecto de las competencias de los participantes. A cada criterio le asignaron una puntuación que osciló entre o,o y 5,o.

Se calculó el índice de concordancia en la revisión de cada ítem (Mandeville, 2005). Este índice expresa en qué medida hubo coincidencia en la clasificación entre los observadores frente al total de elementos examinados. El porcentaje de acuerdo en cuanto a claridad fue de $67 \%$; en redacción, $71 \%$; en información necesaria y suficiente, $69 \%$; y en complejidad adecuada a las competencias, $74 \%$. La escala de 


\begin{tabular}{|c|c|c|}
\hline CUADRO 1 & \multicolumn{2}{|c|}{ Definición de variables e indicadores } \\
\hline Variables & Definición & Indicadores \\
\hline \multirow{3}{*}{ Inclusión } & \multirow{3}{*}{$\begin{array}{l}\text { Estrategias de enseñanza flexibles } \\
\text { e innovadoras que permitan una } \\
\text { educación personalizada, reflejada } \\
\text { en el reconocimiento de estilos de } \\
\text { aprendizaje y capacidades entre los } \\
\text { estudiantes. } \\
\text { Proceso orientado a atender la } \\
\text { diversidad de necesidades de todos } \\
\text { los estudiantes. }\end{array}$} & Participación \\
\hline & & Uso de recursos-herramientas que \\
\hline & & $\begin{array}{l}\text { respondan a las necesidades del } \\
\text { aprendiz }\end{array}$ \\
\hline Calidad & $\begin{array}{l}\text { Relación entre los costos invertidos } \\
\text { y los resultados obtenidos a través de } \\
\text { pruebas estandarizadas nacionales }\end{array}$ & $\begin{array}{l}\text { Objetivos ajustados a expectativas de } \\
\text { la comunidad }\end{array}$ \\
\hline \multirow{4}{*}{ Sociodemográficos } & \multirow{4}{*}{$\begin{array}{l}\text { Características generales de la } \\
\text { población que pueden relacionarse } \\
\text { con la calidad educativa }\end{array}$} & Edad y sexo \\
\hline & & Estrato \\
\hline & & Nivel de estudios \\
\hline & & Años en docencia \\
\hline \multirow{5}{*}{$\begin{array}{l}\text { Prácticas pedagógicas } \\
\text { de los docentes }\end{array}$} & \multirow{5}{*}{$\begin{array}{l}\text { Diseño y ejecución de actividades en } \\
\text { el contexto educativo }\end{array}$} & $\begin{array}{l}\text { Identificación de habilidades e inte- } \\
\text { reses de los estudiantes }\end{array}$ \\
\hline & & $\begin{array}{l}\text { Contenidos ajustados a las necesida- } \\
\text { des e intereses de la comunidad }\end{array}$ \\
\hline & & $\begin{array}{l}\text { Integración de campos de conoci- } \\
\text { miento }\end{array}$ \\
\hline & & Promoción de actividades en equipo \\
\hline & & Evaluación continua y negociada \\
\hline
\end{tabular}

Fuente: elaboración propia.

valoración para los índices de concordancia de Landis y Koch (1977) indica que el grado de acuerdo se considera sustancial si oscila entre $61 \%$ y $80 \%$.

\section{Procedimiento}

La recolección de datos consistió en la visita a los colegios y acompañamiento durante el diligenciamiento de las encuestas para docentes y estudiantes. Se procuró la presencia de uno de los investigadores durante la aplicación, con el fin de garantizar la comprensión de cada uno de los ítems por parte de los participantes. La 
aplicación de la encuesta en cada curso duró 1 hora, aproximadamente. Para efectos de análisis se tomaron en cuenta solo las encuestas que contarán con un mínimo de $70 \%$ de respuestas completas y claras que arrojó la cantidad de participantes mencionada previamente.

Para la computación de las respuestas en la base de datos, las variables se conformaron del promedio de los tres ítems por característica. Es decir, se promediaron los valores obtenidos para cada característica y se obtuvieron medidas continuas con las que se procedió a realizar análisis estadísticos descriptivos; pruebas $t$ para establecer si existen diferencias en los niveles percibidos de inclusión (por modelo); y pruebas de correlación de Pearson para establecer la relación entre la percepción de inclusión y los componentes constitutivos de los modelos pedagógicos. Se usó el software de análisis estadístico Stata 12.

\section{Resultados}

Se presenta aquí el reporte de inclusión de docentes y estudiantes, seguido de la asociación entre prácticas docentes y la inclusión reportada por maestros y estudiantes. Después, se mostrarán los resultados de los análisis de relación entre prácticas e inclusión por modelos para dar respuesta a la pregunta que orienta el estudio.

Con el fin de establecer el nivel de confiabilidad del cuestionario se usó el método splitt-half. Los resultados de esta prueba arrojaron un coeficiente de fiabilidad de 0,78 con la prueba Spearman-Brown, lo que puede ser considerado como buena confiabilidad (George; Mallery, 2003; Kaplan; Saccuzzo, 1982).

De acuerdo con los datos recolectados, los reportes de los docentes en ambos modelos evidencian una media mayor de inclusión que los estudiantes: $\mathrm{M}=4,00$ $(\mathrm{SD}=0,49)$ y $\mathrm{M}=3,73(\mathrm{SD}=0,71)$, respectivamente. Análisis exploratorios gráficos por modelo-indican que los datos del Modelo Educativo Comunitario de Oriente (2) reportan menor dispersión y medianas superiores respecto al modelo de Comunidades de Aprendizaje (1), como se muestra en la figura 1. En este último modelo se ubican los puntajes mínimos y máximos para inclusión. De manera detallada, es posible observar que la mayoría de los reportes de inclusión los docentes del Modelo Educativo Comunitario de Oriente se agrupan entre 3,8 y 4,3, mientras que los puntajes de Comunidades de Aprendizaje se ubican entre 3,2 y 4,5. Los resultados de estas exploraciones sugieren la existencia de diferencias en relación con la implementación de cada uno de los modelos y los niveles de inclusión percibidos por los docentes. 
FIGURA 1 Distribución de puntajes de inclusión por modelo en docentes

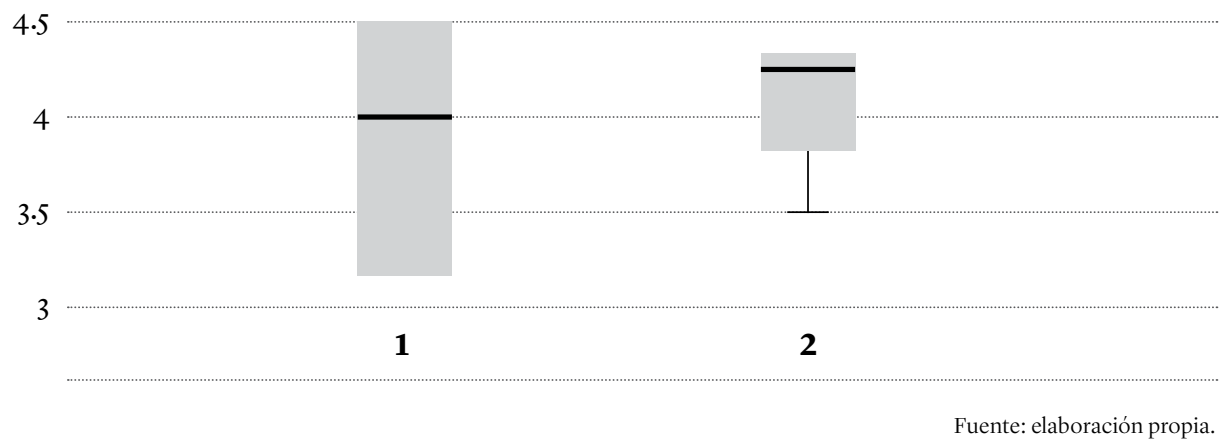

Como se presenta en la figura 2, y de forma contraria a lo encontrado en las distribuciones de datos en los docentes, los reportes de los estudiantes sobre la inclusión en los modelos parecen favorecer a Comunidades de Aprendizaje (1). La mediana fue superior en este último, la mayor concentración de datos se ubicó en puntajes superiores a los del Modelo Educativo Comunitario de Oriente (2) y, además, presentaron menor dispersión. Adicionalmente, los datos mínimos se presentaron en dicho modelo.

FIGURA 2 Distribución de puntajes de inclusión por modelo en estudiantes

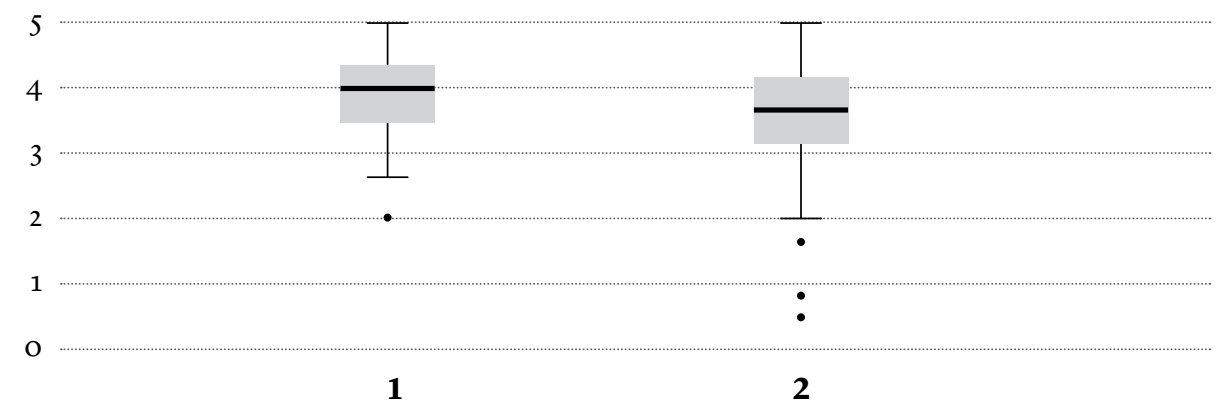

Fuente: elaboración propia. 
Una vez identificada la distribución de puntajes en inclusión por modelo en estudiantes y profesores, y con el fin de conocer cuáles son los aspectos de las prácticas docentes que favorecen la inclusión en cada modelo, se presentan las asociaciones entre los elementos que estructuran la práctica según lo reportado por docentes y estudiantes.

\begin{tabular}{|c|c|c|}
\hline CUADRO 2 & \multicolumn{2}{|c|}{$\begin{array}{l}\text { Relaciones entre prácticas e inclusión en el Modelo Educativo } \\
\text { Comunitario de Oriente - docentes }\end{array}$} \\
\hline \multicolumn{2}{|r|}{ Prácticas } & Inclusión \\
\hline \multirow{2}{*}{\multicolumn{2}{|c|}{ Dialógico }} & $\mathrm{r}=0,98$ \\
\hline & & $\mathrm{p}=0,01^{*}$ \\
\hline \multirow{2}{*}{\multicolumn{2}{|c|}{ Integración de conocimiento }} & $\mathrm{r}=0,98$ \\
\hline & & $\mathrm{p}=\mathrm{o}, \mathrm{o1^{* }}$ \\
\hline \multirow{2}{*}{\multicolumn{2}{|c|}{ Habitancia }} & $r=0,92$ \\
\hline & & $\mathrm{p}=0,07$ \\
\hline \multirow{2}{*}{ Arte } & & $r=0,74$ \\
\hline & & $\mathrm{p}=0,25$ \\
\hline \multirow{2}{*}{\multicolumn{2}{|c|}{ Agentes políticos }} & $r=0,94$ \\
\hline & & $\mathrm{p}=\mathrm{o}, \mathrm{o5}$ \\
\hline \multirow{2}{*}{ TIC } & & $\mathrm{r}=0,51$ \\
\hline & & $\mathrm{p}=\mathrm{o}, 48$ \\
\hline \multirow{2}{*}{\multicolumn{2}{|c|}{ Alineación de objetivos }} & $r=0,97$ \\
\hline & & $\mathrm{p}=\mathrm{o}, \mathrm{o2}^{*}$ \\
\hline \multirow{2}{*}{\multicolumn{2}{|c|}{ Aprendizaje colaborativo }} & $\mathrm{r}=0,85$ \\
\hline & & $p=0,14$ \\
\hline
\end{tabular}

Para el modelo Comunidades de Aprendizaje no se presentaron asociaciones significativas entre las prácticas docentes y la inclusión. Por su parte, como se evidencia en la tabla anterior, en el Modelo Educativo Comunitario de Oriente las practicas docentes que fomentan el conocimiento dialógico, la integración de conocimientos y la alineación de objetivos de aula con los intereses de la comunidad se relacionaron de manera significativa con la inclusión. 
Por su parte, en el caso de los estudiantes, todos los componentes de las prácticas se relacionan positivamente y de manera significativa con la inclusión $(p<0,05)$, aunque a diferentes índices de correlación. La alineación de los objetivos del aula con las necesidades de la comunidad resultó ser el elemento con el índice de correlación más alto $(r=0,60)$, seguido del aprendizaje colaborativo $(r=0,47)$ y de la formación de agentes políticos $(r=0,46)$. El cuadro 3 permite ver estos resultados en detalle.

\section{\begin{tabular}{l|l} 
CUADRO 3 & $\begin{array}{l}\text { Relaciones entre práctica e inclusión en ambos modelos } \\
\text { según estudiantes }\end{array}$
\end{tabular}}

\begin{tabular}{|c|c|c|}
\hline Prácticas & $\begin{array}{c}\text { Comunidades de Apren- } \\
\text { dizaje }\end{array}$ & $\begin{array}{l}\text { Modelo Educativo Comuni- } \\
\text { tario de Oriente }\end{array}$ \\
\hline \multirow{2}{*}{ Dialógico } & $r=0,32$ & $\mathrm{r}=0,49$ \\
\hline & $\mathrm{p}=\mathrm{o}, \mathrm{oo}$ & $\mathrm{p}=\mathrm{o}, \mathrm{oo}$ \\
\hline \multirow{2}{*}{ Integración de conocimiento } & $r=0,35$ & $\mathrm{r}=\mathrm{O}, 46$ \\
\hline & $\mathrm{p}=\mathrm{o}, \mathrm{oo}$ & $p=0, o o$ \\
\hline \multirow{2}{*}{ Habitancia } & $r=0,39$ & $\mathrm{r}=\mathrm{O}, 38$ \\
\hline & $\mathrm{p}=\mathrm{o}, \mathrm{oo}$ & $\mathrm{p}=\mathrm{o}, \mathrm{oo}$ \\
\hline \multirow{2}{*}{ Arte } & $\mathrm{r}=0,28$ & $\mathrm{r}=\mathrm{O}, 33$ \\
\hline & $\mathrm{p}=\mathrm{o}, \mathrm{o} 1$ & $\mathrm{p}=\mathrm{o}, \mathrm{oo}$ \\
\hline \multirow{2}{*}{ Agentes políticos } & $r=0,46$ & $\mathrm{r}=\mathrm{O}, 53$ \\
\hline & $\mathrm{p}=\mathrm{o}, \mathrm{oo}$ & $\mathrm{p}=0,00$ \\
\hline \multirow{2}{*}{ TIC } & $\mathrm{r}=0,22$ & $r=0,42$ \\
\hline & $\mathrm{p}=\mathrm{o}, \mathrm{o} 4$ & $\mathrm{p}=\mathrm{o}, \mathrm{oo}$ \\
\hline \multirow{2}{*}{ Alineación de objetivos } & $r=0,60$ & $\mathrm{r}=0,41$ \\
\hline & $\mathrm{p}=\mathrm{o}, \mathrm{oo}$ & $\mathrm{p}=\mathrm{o}, \mathrm{oo}$ \\
\hline \multirow{2}{*}{ Aprendizaje colaborativo } & $\mathrm{r}=0,47$ & $r=0,45$ \\
\hline & $\mathrm{p}=\mathrm{o}, \mathrm{OO}$ & $\mathrm{p}=\mathrm{o}, \mathrm{oo}$ \\
\hline
\end{tabular}

$\alpha=0,05$

Hasta este punto, se han presentado las relaciones que permiten identificar las prácticas que parecen favorecer la inclusión en las actividades de aula en cada modelo. De acuerdo con esto, y en función de cumplir el objetivo de este estudio, se compararon las medidas de inclusión reportadas por docentes y estudiantes en 
cada uno de los modelos con el fin de establecer diferencias que permitan afirmar mayor efectividad percibida en alguno de estos. A continuación, la tabla 4 presenta los resultados de este análisis.

\begin{tabular}{|l|l|} 
CUADRO 4 & $\begin{array}{l}\text { Diferencias por modelo en los reportes de docentes y estudiantes } \\
\text { sobre inclusión }\end{array}$
\end{tabular}

\begin{tabular}{llll} 
Índices / Grupos & $\begin{array}{c}\text { Comunidades de } \\
\text { aprendizaje }\end{array}$ & $\begin{array}{c}\text { Modelo Educativo } \\
\text { Comunitario de } \\
\text { Oriente }\end{array}$ & Prueba t \\
\hline Docentes & $\mathrm{M}=3,88 \mathrm{SD}=0,67$ & $\mathrm{M}=4,08 \mathrm{SD}=0,19$ & $\mathrm{t}(5)=-0,48 \mathrm{p}=0,64$ \\
\hline Estudiantes & $\mathrm{M}=3,87 \mathrm{SD}=0,58$ & $\mathrm{M}=3,60 \mathrm{SD}=0,83$ & $\mathrm{t}(162)=2,38 \mathrm{p}=0,01^{*}$ \\
\hline
\end{tabular}

Fuente: elaboración propia.

Para el caso de los docentes, se observa en el cuadro 4 que el Modelo Educativo Comunitario de Oriente presenta una media más alta $(4,08)$ que la de Comunidades de Aprendizaje $(3,88)$, aunque esta no resulta estadísticamente significativa. Esto es, los docentes del primer modelo mencionado reportan mayores puntajes de inclusión en su institución. Por otrolado, en el caso de los estudiantes, el modelo Comunidades de Aprendizaje reporta mayor media de puntajes $(3,87)$ que el Modelo Educativo Comunitario de Oriente (3,60), diferencia que resulta estadísticamente significativa.

En síntesis, puede afirmarse que las características asociadas de manera significativa con la inclusión resultaron diferentes para cada muestra y modelo. En el Modelo Educativo Comunitario de Oriente, las prácticas que fomentan el conocimiento dialógico, la integración de conocimientos y la alineación de objetivos se relacionaron de manera significativa con la inclusión, según lo reportado por los docentes. En el de Comunidades de Aprendizaje, los reportes dados por los docentes no arrojan ninguna relación significativa entre algún elemento de la práctica y el establecimiento de la inclusión. Lo anterior no ocurre con los estudiantes encuestados en ambos modelos: según sus reportes, todas las prácticas están relacionadas con niveles de inclusión. Sin embargo, en la comparación de medias de inclusión por modelo, los estudiantes de Comunidades de Aprendizaje reportan mayores puntajes que los del Modelo Educativo Comunitario de Oriente; tal diferencia indicaría, posiblemente, que las prácticas implementadas en Comunidades de Aprendizaje tienen un carácter más inclusivo que las del Modelo Educativo Comunitario de Oriente, al decir de sus aprendices. 


\section{Discusión}

El estudio comentado aquí exploró las prácticas docentes regidas bajo dos modelos pedagógicos alternativos introducidos en Cali como respuesta al sistema educativo tradicional. El objetivo perseguido radicó en identificar los elementos de las prácticas docentes de dos modelos pedagógicos alternativos que pueden fomentar la inclusión en el aula, desde los significados y construcciones de docentes y estudiantes.

Los resultados muestran diferencias significativas entre estudiantes y maestros respecto de los reportes sobre la implementación de cada modelo y su relación con la inclusión, y entre maestros por modelo. Los análisis de los reportes de los maestros de Comunidades de Aprendizaje indican que ninguno de los elementos de las prácticas propuestos por el modelo se asocia de manera significativa con el favorecimiento de la inclusión; por el contrario, en el Modelo Educativo Comunitario de Oriente el análisis arrojó que el conocimiento dialógico, la integración de conocimientos y la alineación de objetivos se relacionaron de manera significativa con la inclusión. Esto podría deberse al sentido mismo que para los docentes tienen estos aspectos centrales en su modelo. Es decir, este modelo plantea de manera central en la construcción de su PEI un cambio de posición, tanto del estudiante como del docente, respecto a la posición que ocupan en la educación tradicional. De esta manera, permitir que el estudiante tome la palabra, asuma una posición respecto al aprendizaje y lo relacione con las vivencias de su entorno (componente dialógico, alineación de objetivos e integración de campos de conocimiento) son aspectos claves a desarrollar de manera curricular por cada docente en su trabajo escolar. La propuesta de integración de conocimientos, a su turno, no pone de relieve el trabajo por áreas de conocimiento, sino que permite una integración de las mismas a la luz de un proyecto transversal por ciclos.

Este cambio de posición que exige el Modelo Educativo Comunitario de Oriente parece ser lo que se evidencia en estos resultados por cuanto muestra cómo los docentes se han enterado de lo que comporta esta apertura a dar un rol activo a sus estudiantes; este solo es posible si ellos mismos, desde el salón de clase y desde lo que organizan en cada ciclo, generan cambios en la planeación. Por supuesto, una vivencia de cambio da cuenta de una toma de conciencia desde este lugar: asílo proponen Echeita y Duck (2008) en cuanto a que lo inclusivo, al comportar cambios en los procesos de enseñanza y de aprendizaje, permite transformaciones en la escuela misma, a la vez que trasforma los roles de los implicados en el proceso educativo, estudiantes, maestros y la comunidad educativa correspondiente. De esta manera, el hecho mismo de que los proyectos por ciclos en el Modelo Comunitario de Oriente incluyan tener en cuenta las situaciones del entorno, así como dar lugar al estudiante 
en relación con su contexto, su historia, sus intereses y necesidades, hace evidente que las prácticas educativas de los maestros tradicionales, en las cuales no se tenía en cuenta el contexto, cambian, ya que no están de antemano organizadas en guías de aprendizaje. Ellos deben crearlas, en tanto no es lo común encontrar prácticas docentes que retomen lo real del contexto y apliquen diferentes dominios de conocimiento en una clase. Este movimiento de un maestro que ostenta un saber y que lo ofrece a sus estudiantes a uno que, a partir de lo que los estudiantes traen desde su entorno y sus intereses, ofrece un conocimiento para crear formas de aprendizaje concertadas, parece ser muy sentido por los docentes; dan cuenta de ello en sus respuestas. Ahora bien, la zona de la ciudad en la que viven los estudiantes de esta institución comporta cierta vulnerabilidad social; tratar de relacionar los conocimientos que tienen que trabajar en las clases con la comprensión del mundo que los rodea, convierte el trabajo educativo en un ejercicio que, en términos cognitivos, exige formas distintas a las usadas tradicionalmente, basadas en la transmisión de conocimiento. La adopción de perspectivas dialógicas permite que, al escuchar a los estudiantes, tenga lugar a la diferencia social, étnica, religiosa, etc.; esto parece corroborarse en gran medida desde la perspectiva de los docentes. En el caso del Modelo Educativo de Oriente, las diferencias son incluidas en los proyectos escolares, como lo propone el PEI de la institución.

Por otro lado, los resultados de los docentes que implementan la estrategia Comunidades de Aprendizaje podrían estar relacionados con el poco tiempo de implementación de la propuesta, mientras que el Modelo Educativo Comunitario de Oriente parece gozar de un mayor reconocimiento entre los docentes. Comunidades de Aprendizaje es una propuesta reciente que podría requerir de un desarrollo mayor para evaluar sus resultados y la percepción de los docentes, sobre todo habida cuenta de las respuestas de los estudiantes, para quienes sí existiría una relación entre la estrategia y la inclusión educativa.

Por otra parte, se encontraron diferencias entre los reportes dados por docentes y estudiantes. El análisis de los reportes de los segundos, por modelo, mostró que todos los componentes de las prácticas se relacionan de manera significativa con la inclusión. Esto podría explicarse en función de que ambas propuestas pedagógicas - tanto el modelo como la estrategia-comportan un cierto aprendizaje activo, que propone un lugar de agentividad para el estudiante. En la estrategia Comunidades de Aprendizaje este punto es central, por cuanto las actuaciones de éxito tienen como eje la posición de dialogicidad entre maestro y estudiante. Este lugar de agentividad es determinante en cuanto a una postura inclusiva, como se encuentra en un análisis realizado en relación con las tertulias dialógicas expuesto en Cantor (2018); allí se evidencia cómo la perspectiva educativa propuesta en la estrategia Comunidades 
de Aprendizaje busca, de forma sistemática, crear un espacio de interacción real que propenda al aprendizaje dialógico.

Por otro lado, el PEI del Modelo Educativo Comunitario de Oriente propone el escenario escolar como un espacio donde lo educativo comporta lo subjetivo; es decir, el trabajo escolar, no es únicamente desde lo epistemológico, desde el conocimiento, y por ello, no debe dejar de lado lo ontológico (Packer, 2016). Desde esta postura, la escuela constituye subjetividades que, si bien se propone que logren asumir los retos de la posmodernidad, lo hagan en función de unos valores de su contexto social y cultural. Para cumplir este objetivo, en la propuesta creada por los docentes y el cuerpo administrativo de esta institución, la escuela no puede estar escindida entre el discurso y la práctica, y evitando de esta forma estar de espaldas a la realidad (Parra, 1996). Por ello, la propuesta de trabajo por ciclos, parte de elementos que relacionen el conocimiento con las preguntas que el contexto real plantea a partir de los proyectos de aula, lo que permite articular la escuela con lo que vive la comunidad. Esto permite ofrecer alternativas de solución de problemas de la comunidad que, al ser resueltos o incluso analizados en los espacios de clase, traen a la realidad escolar el reconocimiento a la diferencia de ideas, culturas, etnias y religiones; esto permite que los contenidos de clase se articulen con las necesidades sentidas por la comunidad y los estudiantes, con lo cual se logra que las propuestas didácticas en sí mismas sean inclusivas.

La concepción de educación que propone esta institución constituye un proyecto gestado por el cuerpo docente de la institución en diálogo con la comunidad (Institución Educativa Nuevo Latir, 2011); su misión es problematizar la realidad para encontrar salidas en las cuales los estudiantes, con sus ideas para resolver los problemas que viven, son agentes de su propia educación. Esta concepción implica que el maestro no está en un lugar de poder jerárquico, sino que ejerce uno más horizontal en relación con la construcción del conocimiento; ello genera procesos de enseñanza-aprendizaje participativos en los que cada estudiante encuentra la oportunidad de desarrollar sus competencias cognitivas, sociales y relacionales. Realizar el trabajo pedagógico de esta forma significa un cambio de rol, del que dan cuenta los estudiantes en la medida en que tienen voz, son incluidos y se sienten activos en su espacio escolar.

La comparación entre las medias de los puntajes de inclusión de maestros y estudiantes mostró que, por una parte, los docentes del Modelo Educativo Comunitario de Oriente perciben mayor inclusión en sus prácticas de aula (en concordancia con las correlaciones encontradas) que los docentes de Comunidades de Aprendizaje. Sin embargo, como una cuestión interesante, los análisis de los mismos datos en los estudiantes muestran una diferencia contraria; mayor media de puntajes para 
Comunidades de aprendizaje que para el Modelo Educativo Comunitario de Oriente, con diferencia significativa. Estos resultados particulares podrían explicarse porque las actuaciones educativas de éxito, que se proponen en la estrategia educativa de Comunidades de Aprendizaje - evaluada a través de las percepciones de los estudiantes-, está centrada de manera muy fuerte en lo dialógico. Los estudiantes no reciben todas las clases bajo la estrategia que propone Comunidades de Aprendizaje, sino que se trata de algunas actividades dentro de la rutina escolar. Por lo regular, y como se observó en las dos instituciones, la estrategia solo se materializaba a través de dos de las actividades educativas de éxito que promueve la estrategia, las tertulias dialógicas y los grupos interactivos. Ambas actividades dejan en clara evidencia el cambio de rol, ya que con este se busca que todos los estudiantes participen en las clases: para ello está construido el dispositivo de la estrategia en sí misma.

La aparición de actividades disruptivas en la cotidianidad de una escuela con actividades escolares tradicionales da lugar a una importante aceptación de los estudiantes. Como lo muestran los resultados, la percepción de los estudiantes califica de forma positiva las actividades y las reconoce como incluyentes, en la medida en que estimulan la participación de ellos y de otros miembros de la comunidad educativa en las actividades del aula. Esto puede considerarse un logro significativo en el proceso de transformación de los entornos escolares en espacios incluyentes. Como se ha visto, la adopción de modelos y estrategias que adoptan perspectivas socioconstructivistas son bien valoradas por los miembros de las comunidades educativas estudiadas: se perciben como inclusivas y promueven la participación activa de los miembros, al sentir reconocidas sus necesidades y voces en un entorno donde tradicionalmente no eran escuchados.

\section{Referencias}

Ainscow, Mel; Booth, Tony; Dyson, Alan (2006). Improving Schools, Developing Inclusion. London: Routledge. https://doi.org/10.4324/9780203967157

Ainscow, Mel; Hopkins, David; Southworth, Geoff; West, Mel (2001). Hacia escuelas eficaces para todos: Manual para la formación de equipos docentes (Vol. 85). Madrid: Narcea Ediciones.

Allman, Dan (2013). The Sociology of Social Inclusion. SAGE Open, 3(1). https://doi. org/10.1177/2158244012471957

Arias, Alexandra (2014). Cuestiones más que conceptuales sobre la inclusión educativa en Colombia. Entramados: educación y sociedad, 1(1), 191-20o. Recuperado de https://fh.mdp. edu.ar/revistas/index.php/entramados/article/view/1090 
Arias, Leydin; Bedoya, Katerine; Benitez, Cristina; Carmona, Julieth; Castaño, Juan Carlos; Castro, Lina...; Villa, Luz Mary (2007). Formación docente. Una propuesta para promover prácticas pedagógicas inclusivas. Educación y Pedagogía, 14(47), 153-162. Recuperado de http://bibliotecadigital.udea.edu.co/handle/10495/3012

Arnaiz, Pilar (2011). Luchando contra la exclusión: buenas prácticas y éxito escolar. Innovación educativa, 21, 23-35. Recuperado de http://www2.escuelascatolicas.es/pedagogico/ Documents/art\%2ode\%2oPilar\%2oArnaiz.pdf

Aubert, Adriana; Flecha, Ainhoa; García, Carme; Flecha, Ramón; Racionero, Sandra (2008). Aprendizaje dialógico en la Sociedad de la Información. Barcelona: Hipatia Editorial.

Beltrán, Yolima; Martínez, Yexica; Vargas, Ángela (2015). El sistema educativo colombiano en el camino hacia la inclusión: Avances y retos. Educación y educadores, 18(1), 62-75. Recuperado de http://www.scielo.org.co/scielo.php?pid=So123-12942015000100004\&script=sci_ abstract\&tlng=pt

Bernstein, Basil (1990). Class, codes and control: the structuring of pedagogic discourse (vol. 4). London: Routledge.

Booth, Tony; Ainscow, Mel (2002). Index for Inclusion: Guía para la evaluación y mejora de la educación inclusiva. Madrid: Consorcio Universitario para la Educación Inclusiva.

Booth, Tony; Nes, Karit; Strømstad, Mary (2003). Developing Inclusive teacher Education. London: Routledge.

Bravo, Patricia; Santos, Ofelia (2019). Percepciones respecto a la atención a la diversidad o inclusión educativa en estudiantes universitarios. Sophia, colección de filosofía de la educación, 26(1), 327-352. https://doi.org/10.17163/soph.n26.2019.10

Burgstahler, Sheryl (2012). Universal Design: Process, Principles, and Applications. Obtenido de University of Washington. DO-IT. Recuperado de https://files.eric.ed.gov/fulltext/ ED506550.pdf

Cantor, Jackeline (2018). Las prácticas inclusivas y la implementación de la tertulia literaria como estrategia pedagógica: tensiones. En Una década del programa de Psicología en la Universidad Icesi. Trayectoriasy enfoques plurales (pp. 79-107), editado por Omar Alejandro Bravo. Cali, Colombia: Universidad Icesi.

Clavijo, Ruth Germania; Bautista, María José (2020). La educación inclusiva. Análisis y reflexiones en la educación superior ecuatoriana. Alteridad, 15(1), 113-124. https://doi. org/10.17163/alt.v15n1.2020.09

Decreto 1421 de 2017 (29 de agosto), por el cual se reglamenta en el marco de la educación inclusiva la atención educativa a la población con discapacidad. Recuperado de http:// es.presidencia.gov.co/normativa/normativa/DECRETO\%201421\%20DEL\%2029\%20 DE\%2OAGOSTO\%2oDE\%202017.pdf 
Díez, Javier; Flecha, Ramón (2010). Comunidades de Aprendizaje: un proyecto de transformación social y educativa. Revista Interuniversitaria de Formación del Profesorado, 24(1), 19-30. Recuperado de https://www.redalyc.org/pdf/274/27419180oo2.pdf

Echeita, Gerardo; Duk, Cynthia (2008). Inclusión Educativa. REICE. Revista Iberoamericana Sobre Calidad, Eficacia Y Cambio En EducacióN, 6(2). Recuperado de https://revistas.uam. es/index.php/reice/article/view/5436/5874

Echeverría, Rebelín; Flores, Mirta (2018). Distancia social, creencias estereotípicas y emociones hacia las personas con discapacidad en universitarios mexicanos: diferencias por sexo. Revista Española de Discapacidad, 6(I), 221-239. Recuperado de https://dialnet.unirioja.es/ servlet/articulo? codigo $=6450116$

Ferguson, Dianne (2008). International trends in inclusive education: The continuing challenge to teach each one and everyone. European Journal of Special Needs Education, 23(2), 109-120. https://doi.org/10.1080/o8856250801946236

Flecha, Ainhoa; García, Rocío; Gómez, Aitor; Latorre, Antonio (2009). Participación en escuelas de éxito: una investigación comunicativa del proyecto Includ-ed. Culturay educación, 21(2), 183-196. https://doi.org/10.1174/113564009788345899

Florian, Lani (2014). La educación especial en la era de la inclusión. ¿El fin de la educación especial o un nuevo comienzo? Revista Latinoamericana de Educación Inclusiva, 7(2), 27-36. Recuperado de http://repositoriocdpd.net:808o/handle/123456789/1705

Fuchs, Douglas; Fuchs, Lynn (1994). Inclusive Schools Movement and the Radicalization of Special Education Reform. Exceptional Children, 6o(4), 294-309. https://doi. org/10.1177/001440299406000402

George, Darren; Mallery, Paul (2003). SPSS for windows step by step: A simple guide and reference. Boston, EE. UU.: Allyn y Bacon.

Guerrero, Catalina (2012). Hacia la construcción de procesos y prácticas "exclusivas": Metodologías para la intervención. Quaderns d'animació i educació social, 16(5). Recuperado de https://dialnet.unirioja.es/servlet/articulo?codigo=7591169

Infante, Marta (2010). Desafíos a la formación docente: inclusión educativa. Estudios Pedagógicos, 36(1), 287-297. Recuperado de https://scielo.conicyt.cl/pdf/estped/v36n1/art16.pdf

Institución Educativa Nuevo Latir (2011). Proyecto Educativo Institucional Nuevo Latir. Recuperado de http://ienuevolatircali.edu.co/site/wp-content/uploads/2020/o1/PEINuevo-Latir-INSERCIÓN-Servicio-Educación-Personas-Adultas-final.pdf

Kaplan, Robert; Saccuzzo, Dennis (1982). Psychological testing: Principles, Applications, and issues. Wadsworth Cengage Learning.

Landis, Richard; Koch, Gary (1977). The measurement of observer agreement for categorical data. Biometrics, 33, 159-174. Recuperado de https://www.jstor.org/stable/2529310?seq=1 
Lewis, Ann; Norwich, Brahm (Eds.) (2004). Special Teaching for Special Children? Pedagogies for Inclusion. Maidenhead, UK: Open University Press.

Ley 1618 de 2013 (27 de febrero), por la cual se establecen las disposiciones para garantizar el pleno ejercicio de los derechos de las personas con discapacidad. Recuperado de https://discapacidadcolombia.com/phocadownloadpap/LEGISLACION/LEY\%2O ESTATUTARIA\%201618\%20DE\%2O2013.pdf

Lipsky, Dorothy; Gartner, Alan (1996). Inclusion, school restructuring, and the remaking of American society. Harvard Educational Review, 66(4), 762-797. https://doi.org/10.17763/ haer.66.4.3686k7x734246430

Mandeville, Peter (2005). El coeficiente de correlación intraclase. Ciencia UANL, 8(3), 414-416. Recuperado de https://www.redalyc.org/pdf/402/40280322.pdf

Mateus, Luz Eliana; Vallejo, Diana Marcela; Obando, Diana; Fonseca, Laura (2017). Percepción de las prácticas y de la cultura inclusiva en una comunidad escolar. Avances en Psicología Latinoamericana, 35(1), 177-191. https://doi.org/10.12804/revistas.urosario.edu.co/ apl/a.4854

Ministerio de Educación Nacional - MEN (2009). Educación inclusiva con calidad "Construyendo capacidad institucional para la atención a la diversidad”. Guía. Bogotá: Ministerio de Educación Nacional de Colombia.

Packer, Martin (2006). Ontología de la escolaridad. Grupo Cultura y Desarrollo Humano Universidad del Valle. Recuperado de https://psicologiacultural.org/Pdfs/Traducciones/ Ontologia\%2ode\%2ola\%2oescolaridad.pdf

Parra, Rodrigo (1996). Escuela y Modernidad en Colombia. Bogotá: Fundación FES.

Sánchez, José-Eduardo (2018). Características e implicaciones relacionales de dos propuestas innovadoras en educación. En Una Década del programa de Psicología en la Universidad Icesi. Trayectorias y enfoques plurales (pp. 59-77), editado por Omar Alejandro Bravo. Cali: Editorial Universidad Icesi.

Unesco (2008). Inclusive education: The way of the future. En Conclusions and recommendations of the 48th session of the International Conference on Education, (pp. 25-28).

Unesco (2017). Guía para asegurar la inclusión y la equidad en educación. Unesco. http://unesdoc. unesco.org/images/oo25/002595/259592s.pdf

Valdés, René; López, Verónica; Jiménez, Felipe (2019). Inclusión Educativa en relación con la cultura y la convivencia escolar. Educación y Educadores, 22(2), 187-211. https://doi. org/10.5294/edu.2019.22.2.2

Vrasmas, Ecaterina (2018). For a pedagogy of inclusion. A brief overview of the current research on inclusive education. Bulletin of the Transilvania University of Braşov, 11(6o), 3144. Recuperado de http://webbut.unitbv.ro/bulletin/Series\%2OVII/2018/BULETIN\%2O I/o8_Vrasmas-E.pdf 